\title{
Harmonic Variation of Edge Size in Meshing CAD Geometries from IGES Format
}

\author{
Maharavo Randrianarivony \\ Institute of Computer Science \\ Christian-Albrecht University of Kiel, 24098 Kiel, Germany \\ maharavo@informatik.tu-chemnitz.de
}

\begin{abstract}
We shall describe a mesh generation technique on a closed CAD surface composed of a few parametric surfaces. The edge size function is a fundamental entity in order to be able to apply the process of generalized Delaunay triangulation with respect to the first fundamental form. Unfortunately, the edge size function is not known a-priori in general. We describe an approach which invokes the Laplace-Beltrami operator to determine it. We will discuss theoretically the functionality of our methods. Our approach is illustrated by numerical results from the harmonicity of triangulations of some CAD objects. The IGES format is used in order to acquire the initial geometries.
\end{abstract}

Keywords: Geometric modeling, IGES, mesh generation, CAD models, edge size, Delaunay.

\section{Introduction}

The importance of meshes in computer graphics and geometric modeling has become evident in the past decades [1, [6], [8]. In this paper, we address the problem of creating a mesh [7] on a surface $\Gamma$ of a CAD model. We report on the results of our intensive implementation using IGES (Initial Graphics Exchange Specification) format as CAD exchange. Our main goal focus on the fact that we want the variation of lengths of the neighboring edges to be smooth. That will result in meshes composed of nicely shaped triangles. The generation of a surface mesh by means of the generalized Delaunay technique [4, 6] with respect to the first fundamental form 2] requires the knowledge of the edge size function which is unfortunately unknown a-priori [10].

In [1, the authors triangulate planar domains using the Delaunay technique. A method for evaluating the quality of a mesh is given in [6]. Some upper bound of the Delaunay triangulation has been investigated in [4. In this document, our main contribution is the determination of the edge size function for the Delaunay triangulation with respect to the first fundamental form. Additionally, we examine theoretically the functionality of our method. Furthermore, our description is supported by numerical results produced from real IGES data where we investigate mesh harmonicity.

In the next section, we will state our problem more specifically and we will introduce various important definitions. After quickly giving a motivation for

M. Bubak et al. (Eds.): ICCS 2008, Part II, LNCS 5102, pp. 56-65, 2008.

(C) Springer-Verlag Berlin Heidelberg 2008 
planar problems in section 3, we will detail the meshing of a single parametric surface by using generalization of Delaunay triangulation in section 4 . Since the edge size function is known on the boundary, the treatment of the LaplaceBeltrami problem becomes a boundary value problem which we propose to solve numerically in section 5 which contains also the theoretical background of the mesh generation approach. In section 6, we focus on practical aspect and intuitive description for practitioners. Toward the end of the paper, we report on some benchmarks of CAD objects from IGES files and we compare our method with other approaches.

\section{Definitions and Problem Setting}

In our approach, the input is a CAD object which is bounded by a closed surface $\Gamma$ that is composed of $n$ parametric surfaces $\left\{\mathbf{S}_{k}\right\}_{k=1}^{n}$. Each $\mathbf{S}_{k}$ is given as the image of a multiply connected domain $\mathbf{D}_{k} \subset \mathbf{R}^{2}$ by the following function

$$
\mathbf{x}_{k}:\left(u_{1}, u_{2}\right) \in \mathbf{R}^{2} \longrightarrow\left(x_{k, 1}\left(u_{1}, u_{2}\right), x_{k, 2}\left(u_{1}, u_{2}\right), x_{k, 3}\left(u_{1}, u_{2}\right)\right) \in \mathbf{R}^{3}
$$

which is supposed to be bijective and sufficiently smooth [5]. The surfaces $\mathbf{S}_{k}$ will be referred to as the patches of the whole surface $\Gamma$. Every patch of the surface $\Gamma$ is bounded by a list of curves $\mathbf{C}_{i}$. The CAD models come from IGES format where the most important nontrivial entities are enlisted in Table 1 .

Table 1. Most important IGES entities

\begin{tabular}{lcl}
\hline IGES Entities & ID numbers IGES-codes \\
\hline Line & 110 & LINE \\
Circular arc & 100 & ARC \\
Polynomial/rational B-spline curve & 126 & B_SPLINE \\
Composite curve & 102 & CCURVE \\
Surface of revolution & 120 & SREV \\
Tabulated cylinder & 122 & TCYL \\
Polynomial/rational B-spline surface & 128 & SPLSURF \\
Trimmed parametric surface & 144 & TRM_SRF \\
Transformation matrix & 124 & XFORM \\
\hline
\end{tabular}

A mesh $\mathbf{M}_{h}$ is a set of triangles $T_{k} \subset \mathbf{R}^{d}(d=2,3)$ such that the intersection of two nondisjoint different triangles is either a single node or a complete edge. If $d$ is 2 (resp. 3), then we will call $\mathbf{M}_{h}$ a $2 \mathrm{D}$ (resp. 3D) mesh. For a node $A$ in a mesh $\mathbf{M}_{h}$, its valence $\eta(A)$ is the number of edges which are incident upon $A$. The set of nodes which are the endpoints of edges incident upon $A$, and which are different from $A$, will be denoted by $\nu(A)$. Our objective is to generate a $3 \mathrm{D}$ mesh $\mathbf{M}_{h}$ such that all nodes of $\mathbf{M}_{h}$ are located on the surface $\Gamma$. Additionally, we want that the edge lengths vary slowly implying that the lengths of the three edges in any triangle $T \in \mathbf{M}_{h}$ are proportional. 
We will need the matrix $I\left(\mathbf{x}_{k}\right):=\left[g_{i j}\left(\mathbf{x}_{k}\right)\right]$ which represents the first fundamental form where

$$
g_{i j}\left(\mathbf{x}_{k}\right):=<\frac{\partial \mathbf{x}_{k}}{\partial u_{i}}, \frac{\partial \mathbf{x}_{k}}{\partial u_{j}}>=\sum_{p=1}^{3} \frac{\partial x_{k, p}}{\partial u_{i}} \frac{\partial x_{k, p}}{\partial u_{j}} \quad i, j \in\{1,2\} .
$$

\section{Motivation for the Planar Case}

In this section, we want to treat briefly the mesh generation problem in the planar case (see Fig. 1) that should provide both motivation and intuitive ideas which facilitate the description of the general case of parametric surfaces. For that matter, we want to triangulate a planar multiply connected domain $\Omega_{h} \subset \mathbf{R}^{2}$ with polygonal boundaries $P_{h}$. Note that the boundary edge sizes are generally nonuniform (see Fig. 1). That is usually caused by adaptive discretization of some original curved boundaries $P$ according to some error criteria. In order to

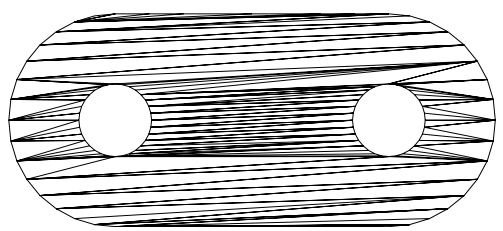

(a)

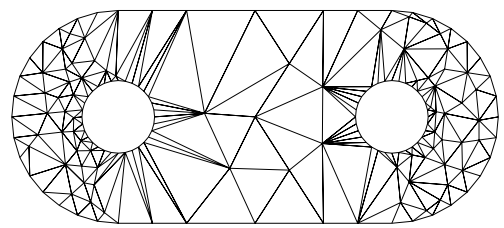

(c)

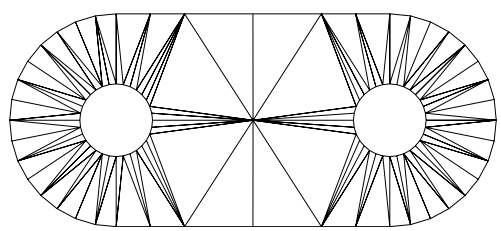

(b)

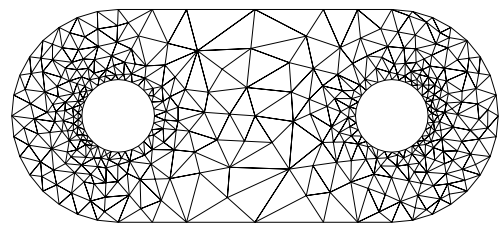

(d)

Fig. 1. Selected steps in mesh recursive refinement

obtain a few of triangles while keeping their good quality shape (Fig. 1(d), the variation of sizes of neighboring edges should be small. Let us introduce the edge size function

$$
\rho: \Omega_{h} \longrightarrow \mathbf{R}^{+}
$$

If this function is explicitly known, then a way to obtain the mesh is to start from a very coarse mesh (Fig. 1(a) and to apply node insertion [2] in the middle of every edge $[\mathbf{a}, \mathbf{b}]$ whose length exceeds the value of $\rho$ at the midnode of $[\mathbf{a}, \mathbf{b}]$. Delaunay edge flipping is simultaneously applied to achieve better angle conditions. Unfortunately, the value of $\rho$ is not known in practice. Since the edge 
size function $\rho$ is known at the boundaries $\partial \Omega_{h}=P_{h}$, we consider the following boundary value problem:

$$
\Delta \rho:=\frac{\partial^{2} \rho}{\partial u_{1}^{2}}+\frac{\partial^{2} \rho}{\partial u_{2}^{2}}=0 \quad \text { in } \quad \Omega_{h},
$$

with the nonhomogeneous Dirichlet boundary condition given by the edge sizes at the boundary. That means the edge size function is required to be harmonic. A harmonic function satisfies in general the mean value property:

$$
\rho\left(a_{1}, a_{2}\right)=\frac{1}{2 \pi} \int_{0}^{2 \pi} \rho\left(a_{1}+r \cos \theta, a_{2}+r \sin \theta\right) d \theta .
$$

That is, $\rho(\mathbf{a})$ is ideally the same as the average of the values of $\rho$ in a circle centered at $\mathbf{a}=\left(a_{1}, a_{2}\right)$. In that way, the edge size function $\rho$ has practically small variation. Meshes with small edge size variation have advantages [10] in graphics and numerics. First, they use few triangles while not loosing shape quality. Second, they prevent numerical instabilities which are not desired in simulations.

\section{Meshing Using the First Fundamental Form}

In this section, we summarize the meshing of a single patch $\mathbf{S}_{k}$ specified by the smooth parametric function $\mathbf{x}_{k}$ given in (1). To simplify the notation, we will drop the index $k$ in the sequel. The approach in triangulating $\mathbf{S}$ is processed in two steps. First, a 2D mesh on the parameter domain $\mathbf{D}$ is generated according to the first fundamental form. Afterwards, the resulting $2 \mathrm{D}$ mesh is lifted to the parametric surface $\mathbf{S}$ by computing its image by $\mathbf{x}$. We will call an edge of a mesh in the parameter domain a $2 D$ edge and an edge in the lifted mesh a $3 D$ edge. For that purpose, one starts from a coarse 2D mesh of $\mathbf{D}$ and a generalized two dimensional Delaunay refinement is used as summarized below. Similarly to the planar case, we introduce an edge size function $\rho$ which is defined now on the parametric surface $\rho: \mathbf{S} \longrightarrow \mathbf{R}^{+}$. By composing $\rho$ with the parameterization $\mathbf{x}$ of $\mathbf{S}$, we have another function $\tilde{\rho}:=\rho \circ \mathbf{x}$ which we will call henceforth "parameter edge size function" because it is defined for all $\mathbf{u}=(u, v)$ in the parameter domain. Let us consider a $2 \mathrm{D}$ edge $[\mathbf{a}, \mathbf{b}] \subset \mathbf{D}$ and let us denote the first fundamental forms at $\mathbf{a}$ and $\mathbf{b}$ by $I_{\mathbf{a}}$ and $I_{\mathbf{b}}$ respectively. Further, we introduce the following average distance between $\mathbf{a}$ and $\mathbf{b}$

$$
d_{\text {Riem }}(\mathbf{a}, \mathbf{b}):=\sqrt{\overrightarrow{\mathbf{a b}}^{T} T \overrightarrow{\mathbf{a b}}} \quad T:=0.5\left(I_{\mathbf{a}}+I_{\mathbf{b}}\right) .
$$

The $2 \mathrm{D}$ edge $[\mathbf{a}, \mathbf{b}]$ is split if this average distance exceeds the value of the parameter edge size function $\tilde{\rho}$ at the midnode of $[\mathbf{a}, \mathbf{b}]$. Note that no new boundary nodes are introduced during that refinement because only internal edges are allowed to be split. Consider now a $2 \mathrm{D}$ edge $[\mathbf{a}, \mathbf{c}]$ shared by two triangles which form a convex quadrilateral $[\mathbf{a}, \mathbf{b}, \mathbf{c}, \mathbf{d}]$. Denote by $T$ the average values of the 
first fundamental forms $I_{\mathbf{a}}, I_{\mathbf{b}}, I_{\mathbf{c}}$ and $I_{\mathbf{d}}$ at those nodes. The edge $[\mathbf{a}, \mathbf{c}]$ is flipped into $[\mathbf{b}, \mathbf{d}]$ if the next generalized Delaunay angle criterion is met

$$
\|\overrightarrow{\mathbf{b c}} \times \overrightarrow{\mathbf{b a}}\|\left(\overrightarrow{\mathbf{d a}}^{T} T \overrightarrow{\mathbf{d c}}\right)<\|\overrightarrow{\mathbf{d a}} \times \overrightarrow{\mathbf{d c}}\|\left(\overrightarrow{\mathbf{c b}}^{T} T \overrightarrow{\mathbf{b a}}\right)
$$

We would like now to describe the procedure of obtaining the initial coarse triangulation. Suppose that we have a $2 \mathrm{D}$ domain $\mathbf{P}$ which may contain some holes and which has polygonal boundaries. We may think of $\mathbf{P}$ as a polygonal discretization of the parameter domain $\mathbf{D}$. First, the polygon $\mathbf{P}$ is split into a few simply connected polygons $\mathbf{P}=\bigcup_{i=1}^{N} \mathbf{P}^{(i)}$. Afterwards, we do the following for every simply connected polygon $\mathbf{P}^{(i)}$. One initializes its set of triangles as empty set $\mathcal{T}_{h}^{(i)}=\emptyset$. Then, one finds a triangle $T$ which can be chopped off from $\mathbf{P}^{(i)}$. We apply some updating $\mathbf{P}^{(i)}:=\mathbf{P}^{(i)} \backslash T$ and $\mathcal{T}_{h}^{(i)}:=\mathcal{T}_{h}^{(i)} \cup T$. We repeat the same chopping until $\mathbf{P}^{i}$ has no vertices left. Finally, the triangulation of $\mathbf{P}$ is the union of all triangulations: $\mathcal{T}_{h}:=\cup_{i=1}^{N} \mathcal{T}_{h}^{(i)}$.

\section{Edge Size and Theoretical Discussion}

Let us consider a parametric surface $\mathbf{S}$ and a differentiable function $F: \mathbf{S} \longrightarrow \mathbf{R}$. The Laplace-Beltrami operator is defined by

$$
\Delta_{\mathbf{S}} F=-\frac{1}{\sqrt{g}} \frac{\partial}{\partial u_{j}}\left(\sqrt{g} g_{i j} \frac{\partial F}{\partial u_{i}}\right)
$$

in which we use Einstein notation in indexing and $g$ is the determinant of $I$ which we introduced in (2). The function $F$ is said to be harmonic if $\Delta_{\mathbf{S}} F=0$. Since the edge size function $\rho$ should be harmonic, we have the following problem

$$
\left\{\begin{aligned}
-\Delta_{S} \rho & =0 & & \text { in } \mathbf{S} \\
\rho & =\rho_{\text {bound }} & & \text { on } \partial \mathbf{S} .
\end{aligned}\right.
$$

The values of $\rho$ at the boundary which are denoted by $\rho_{\text {bound }}$ are known because they are described by the boundary discretization. We will sketch in this section how to numerically solve the boundary value problem (9) by means of the finite element method. For that end, we take a temporary mesh $\mathbf{M}_{h}$ on $\mathbf{S}$ and we denote its boundary by $\partial \mathbf{M}_{h}$. For a smooth function $\phi$ which takes value zero at the boundary we have

$$
-\int_{\mathbf{S}} \Delta_{\mathbf{S}} \rho \phi=\int_{\mathbf{S}}<\nabla_{\mathbf{S}} \rho, \nabla_{\mathbf{S}} \phi>=: a(\rho, \phi) .
$$

Let us define the following set of approximating linear space $V_{h}:=\left\{f \in \mathbf{C}^{0}\left(\mathbf{M}_{h}\right)\right.$ : $\left.f_{\mid T} \in \mathbf{P}_{1} \forall T \in \mathbf{M}_{h}\right\}$, where $\mathbf{C}^{0}\left(\mathbf{M}_{h}\right)$ denotes the space of functions which are globally continuous on $\mathbf{M}_{h}$ and $\mathbf{P}_{1}$ the space of linear polynomials. For a function $g$ we define the set $V_{h}^{g}:=\left\{f \in V_{h}: f=g\right.$ on $\left.\partial \mathbf{M}_{h}\right\}$ which is not in general a linear space. The approximated solution $\rho_{h}$ will reside in the set $V_{h}^{\rho_{\text {bound }}}$. In order 
to find $\rho_{h}$, we pick an element $\tilde{\rho}$ of $V_{h}^{\rho_{\text {bound }}}$ and define $\mu_{h}$ by setting $\rho_{h}=\tilde{\rho}+\mu_{h}$. The function $\rho_{h}$ is therefore completely determined if we know the new unknown function $\mu_{h}$ which resides interestingly in $V_{h}^{0}$. Observe that $V_{h}^{0}$ is a linear space in which we choose a basis $\left\{\phi_{i}\right\}_{i \in I}$. As a consequence, the function $\mu_{h}$ is a linear combination of $\left\{\phi_{i}\right\}_{i \in I}: \mu_{h}=\sum_{i \in I} \mu_{i} \phi_{i}$. By introducing the following bilinear form $a_{h}(\cdot, \cdot)$

$$
a_{h}(\psi, \phi):=\sum_{T \in \mathbf{M}_{h}} a_{T}(\psi, \phi) \quad \text { with } \quad a_{T}(\psi, \phi):=<\nabla_{T} \psi, \nabla_{T} \phi>,
$$

we have $a_{h}\left(\rho_{h}, \phi\right)=0 \quad \forall \phi \in V_{h}^{0}$ or equivalently $a_{h}\left(\mu_{h}, \phi\right)=-a_{h}(\tilde{\rho}, \phi)$ $\forall \phi \in V_{h}^{0}$. Since $\phi_{i}$ builds a basis for $V_{h}^{0}$, this leads to a linear equation

$$
\sum_{i \in I} a_{h}\left(\phi_{i}, \phi_{j}\right) \mu_{i}=-a_{h}\left(\tilde{\rho}, \phi_{j}\right) \quad \forall j \in I .
$$

One can assemble the stiffness matrix $M_{i j}:=a_{h}\left(\phi_{i}, \phi_{j}\right)$ and solve (12) for $\mu_{i}$ which yields the value of $\mu_{h}$. For every triangle $T$ in $\mathbf{M}_{h}$ with internal angles $\alpha_{1}, \alpha_{2}$ and $\alpha_{3}$, its contribution to the stiffness matrix $M$ is

$$
M_{T}=0.5\left[\begin{array}{lcr}
\cot \alpha_{2}+\cot \alpha_{3} & -\cot \alpha_{3} & -\cot \alpha_{2} \\
-\cot \alpha_{3} & \cot \alpha_{1}+\cot \alpha_{3} & -\cot \alpha_{1} \\
-\cot \alpha_{2} & -\cot \alpha_{1} & \cot \alpha_{1}+\cot \alpha_{2}
\end{array}\right] .
$$

We want now to theoretically discuss the applicability of the former triangulation to a given $\mathrm{CAD}$ model. We will only emphasize on the main points but the complete detail can be seen in [10. The main critical aspects which we want to clarify are twofold. First, it has been shown that from every simply connected [9] polygon $\mathbf{P}$, one may remove two triangles $T_{1}$ and $T_{2}$ (called ears) by introducing internal cuts. Thus, if we suppose that the polygon has $n$ vertices, we need to chop off triangles $(n-2)$ times in order to obtain the initial coarse mesh. As a consequence, a simply connected polygon can be triangulated by using only boundary nodes. For triangulation of multiply connected polygons, we need to split them first into several simply connected polygons [9], 10].

Now, we will show that the linear system from (12) is uniquely solvable. Consider a triangle $T=[A, B, C]$ of the mesh $\mathbf{M}_{h}$. Let $\boldsymbol{N}_{3}$ be the unit normal vector of $T$. Generate two unit vectors $\boldsymbol{N}_{1}$ and $\boldsymbol{N}_{2}$ perpendicular to $\boldsymbol{N}_{3}$ such that $\left(\boldsymbol{N}_{1}, \boldsymbol{N}_{2}, \boldsymbol{N}_{3}\right)$ is an orthonormal system which can be centered at $A$. Since the triangle $T$ is located in the plane spanned by $\left(\boldsymbol{N}_{1}, \boldsymbol{N}_{2}\right)$, every point $\mathbf{x} \in T$ can be identified by $\left(v_{1}, v_{2}\right) \in \mathbf{R}^{2}$ such that $\overrightarrow{A \mathbf{x}}=v_{1} \boldsymbol{N}_{1}+v_{2} \boldsymbol{N}_{2}$.

Consider the triangle $t:=[(0,0),(1,0),(0,1)]$ and let $\varphi$ be the parameterization which transforms $t$ into $T$ :

$$
\left[\begin{array}{l}
\varphi_{1}\left(u_{1}, u_{2}\right) \\
\varphi_{2}\left(u_{1}, u_{2}\right)
\end{array}\right]:=\left[\begin{array}{ll}
V_{1} & W_{1} \\
V_{2} & W_{2}
\end{array}\right]\left[\begin{array}{l}
u_{1} \\
u_{2}
\end{array}\right]
$$

where $\left(V_{1}, V_{2}\right)$ and $\left(W_{1}, W_{2}\right)$ are the components of $W:=\overrightarrow{A B}$ and $V:=\overrightarrow{A C}$ in $\left(\boldsymbol{N}_{1}, \boldsymbol{N}_{2}\right)$. Denote by $M$ the above matrix and let $\theta$ be the inverse of $\varphi$ :

$$
\left[\begin{array}{l}
\theta_{1}\left(v_{1}, v_{2}\right) \\
\theta_{2}\left(v_{1}, v_{2}\right)
\end{array}\right]=\frac{1}{\operatorname{det} M}\left[\begin{array}{cc}
W_{2} & -W_{1} \\
-V_{2} & V_{1}
\end{array}\right]\left[\begin{array}{l}
v_{1} \\
v_{2}
\end{array}\right]
$$


Let $\psi$ be the linear polynomial which transforms $\psi(0,0)=\mu(A), \psi(1,0)=\mu(B)$, $\psi(0,1)=\mu(C)$. Its exact expression is $\psi\left(u_{1}, u_{2}\right)=[\mu(B)-\mu(A)] u_{1}+[\mu(C)-$ $\mu(A)] u_{2}+\mu(A)$. We want to compute $a_{T}(\cdot, \cdot)$ in terms of $\left(u_{1}, u_{2}\right)$. By introducing $a_{i j}:=\partial_{v_{j}} \theta_{i}$, the integrand for $a_{T}(\cdot, \cdot)$ involves $I\left(u_{1}, u_{2}\right):=\left(a_{11} \partial_{u_{1}} \psi+\right.$ $\left.a_{21} \partial_{u_{2}} \psi\right)^{2}+\left(a_{12} \partial_{u_{1}} \psi+a_{22} \partial_{u_{2}} \psi\right)^{2}$. Because of (15), we have $a_{11}=W_{2} / D, a_{12}=$ $-W_{1} / D, a_{21}=-V_{2} / D$ and $a_{22}=V_{1} / D$ where $D=\operatorname{det} M$. By using the fact that $\cos \alpha=<V, W>/(\|V\| \cdot\|W\|)$ and $\sin \alpha=\operatorname{det} M /(\|V\| \cdot\|W\|)$, we obtain $I\left(u_{1}, u_{2}\right)=\frac{1}{D}\left\{[\mu(B)-\mu(C)]^{2} \cot \alpha+[\mu(C)-\mu(A)]^{2} \cot \beta+[\mu(B)-\mu(A)]^{2} \cot \gamma\right\}$. We have therefore

$$
\begin{aligned}
a_{T}(\mu, \mu)= & \int_{t} I\left(u_{1}, u_{2}\right)(\operatorname{det} M) d u_{1} d u_{2} \\
= & 0.5\left\{[\mu(B)-\mu(C)]^{2} \cot \alpha+[\mu(C)-\mu(A)]^{2} \cot \beta+\right. \\
& {\left.[\mu(B)-\mu(A)]^{2} \cot \gamma\right\} . }
\end{aligned}
$$

By introducing $\Psi(\mu):=a_{T}(\mu, \mu)$, the system in (13) can be obtained by $a_{T}(\mu, \nu)=$ $0.5[\Psi(\mu+\nu)-\Psi(\mu)-\Psi(\nu)]$. Denote by $\tilde{W}:=\frac{1}{D}\left(\partial_{u_{1}} \psi\right) W$ and $\tilde{V}:=\frac{1}{D}\left(\partial_{u_{2}} \psi\right) V$, we have $I\left(u_{1}, u_{2}\right)=\|\tilde{W}\|^{2}-2<\tilde{W}, \tilde{V}>+\|\tilde{V}\|^{2}$. Thus, $I\left(u_{1}, u_{2}\right)=0$ iff $\tilde{W}=\lambda \tilde{V}$ for some $\lambda \in \mathbf{R}$. Since $\mu \in V_{h}^{0}$ is globally continuous and it takes zero values at the boundary, we have $\mu=0$. The form $a_{h}(\cdot, \cdot)$ is thus symmetric positive definite. Hence, the linear system from (12) is solvable and the edge size function can be deduced.

\section{Practical Aspect and Intuitive Description}

In this section, we describe a realistic approach which is interesting for practitioners. Intuitively, our meshing approach is similar to the standard 2D Delaunay but we replace the usual Euclidian distance by the one given in (6). That means, the $2 \mathrm{D}$ parameter mesh which might be very anisotropic corresponds to a surface mesh that is very well shaped. In the previous discussions, the triangulation of a single trimmed surface [3] was provided. In practice, an IGES file contains several trimmed surfaces. Therefore, we will describe briefly how to triangulate the whole surface $\Gamma$ composed of the patches $\mathbf{S}_{1}, \cdots, \mathbf{S}_{n}$. First, we discretize (Fig. 2) the curved boundaries $\mathbf{C}_{i}$ by piecewise linear curves $\tilde{\mathbf{C}}_{i}$ in which we aim at both accuracy and smoothness: curves which are almost straight need few vertices while those having sharp curvatures need many vertices 10. Afterwards, we map the $3 \mathrm{D}$ nodes of the relevant piecewise linear curves $\tilde{\mathbf{C}}_{i}$ back to the parameter domain $\mathbf{D}_{k} \subset \mathbf{R}^{2}$ for each patch $\mathbf{S}_{k}$. Thus, we may apply the former approach to the polygons formed by the $2 \mathrm{D}$ preimages of the $3 \mathrm{D}$ nodes. In other words, a mesh $\mathbf{M}_{k}$ is created by using the technique in section 4 for each surface patch $\mathbf{S}_{k}$. Finally, one merges the meshes $\mathbf{M}_{1}, \cdots, \mathbf{M}_{n}$ in order to obtain the final mesh of $\Gamma$. Since we use no boundary nodes other than those corresponding to the preimages, no new nodes are inserted during the refinements. As a consequence, nodes at the interface of two adjacent patches align. 


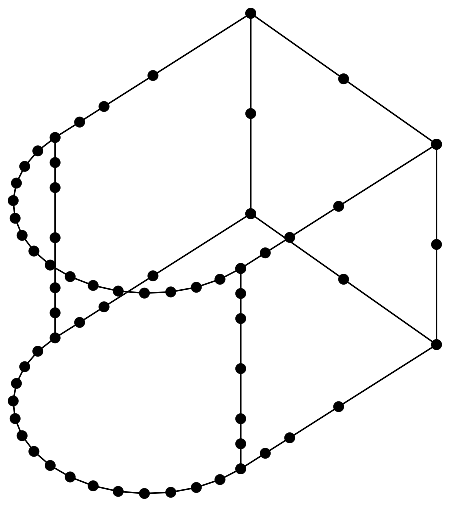

(a)

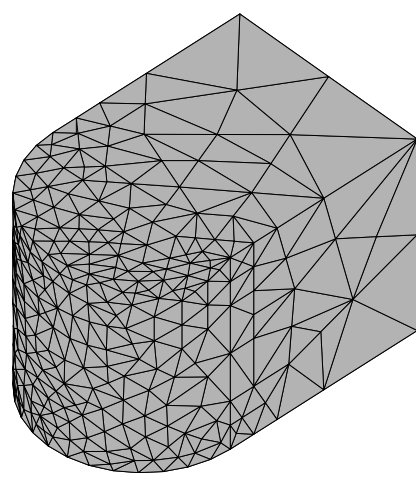

(b)

Fig. 2. (a)Boundary nodes (b)Surface mesh

\section{Numerical Results and Comparison}

In this section, we illustrate our former approach numerically. Additionally, we compare our results with other meshing approaches. The CAD objects whose surfaces have to be triangulated are given as input in IGES files. We consider four CAD objects which have respectively 30, 25, 24 and 26 patches. We used the former method to generate meshes on their surfaces. The resulting meshes, having respectively 11834, 7944, 7672 and 8932 elements, as portrayed in Fig. 3. We would like to investigate the harmonicity of the meshes which we want to define now. For any considered node $A \in \mathbf{R}^{3}$ of a mesh $\mathbf{M}_{h}$, we define

$$
\rho(A):=\frac{1}{\eta(A)} \sum_{B \in \nu(A)}\|\overrightarrow{A B}\|
$$

to be the average edge length. Now we define $r(A)$ to be the length of the shortest edge incident to a node $A$ and we let $s_{i}(A)$ be the intersection of the $i$-th edge $\left[A, B_{i}\right]$ incident upon $A$ and the sphere centered at $A$ with radius $r(A)$. We define the discrete mean value $\rho_{\text {mean }}(A)$ to be

$$
\rho_{\text {mean }}(A):=\frac{1}{\eta(A)} \sum_{i=1}^{\eta(A)} \rho\left(s_{i}(A)\right)
$$

in which $\rho\left(s_{i}(A)\right)$ is the following convex combination of $\rho(A)$ and $\rho\left(B_{i}\right)$

$$
\rho\left(s_{i}(A)\right):=\frac{\left\|\overrightarrow{A s_{i}}\right\|}{\left\|\overrightarrow{A B_{i}}\right\|} \rho\left(B_{i}\right)+\left(1+\frac{\left\|\overrightarrow{A s}_{i}\right\|}{\left\|\overrightarrow{A B_{i}}\right\|}\right) \rho(A) .
$$




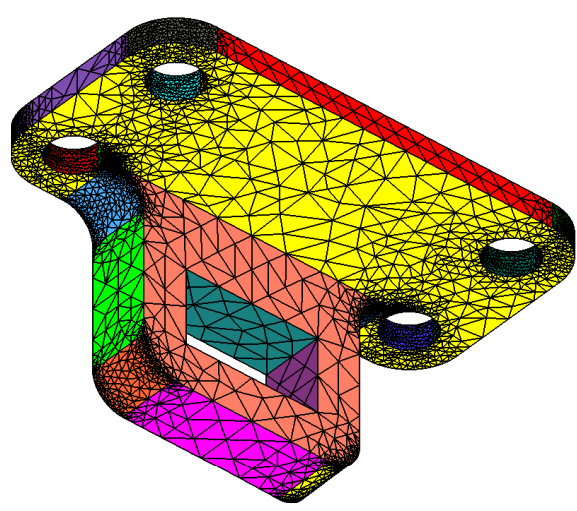

(a)

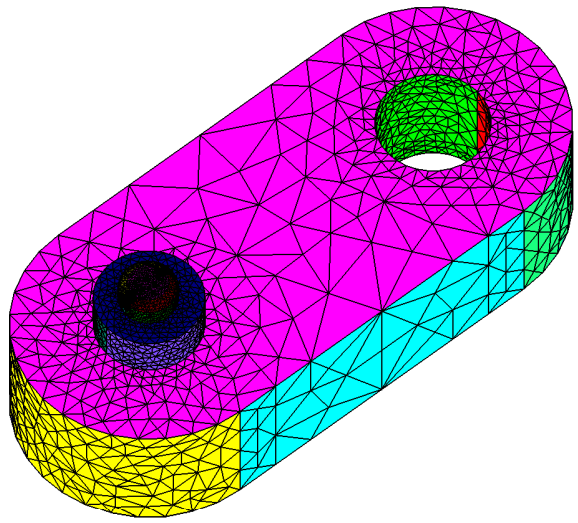

(c)

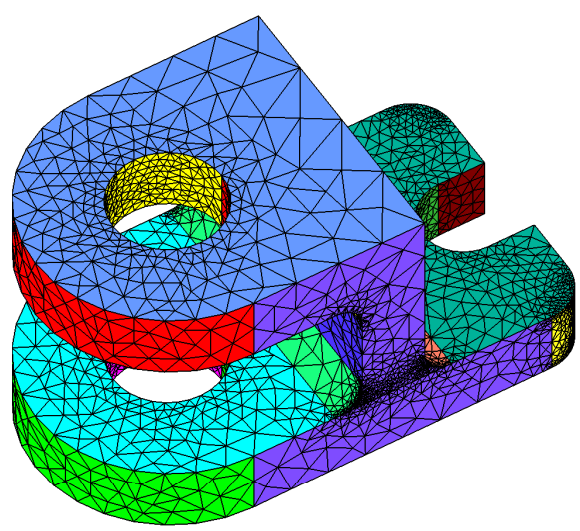

(b)

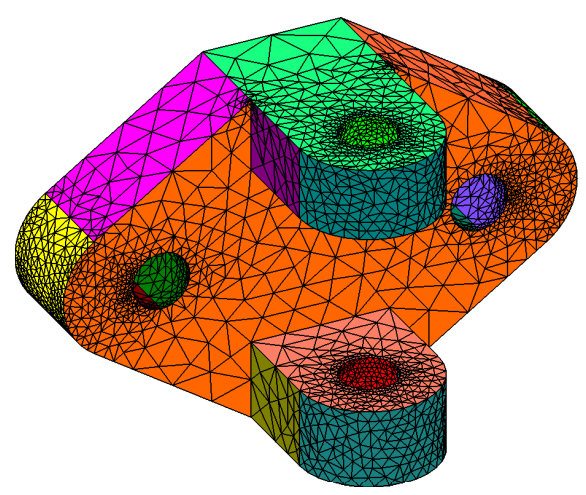

(d)

Fig. 3. Four meshes generated from CAD objects

Table 2. Harmonicity of the four meshes

\begin{tabular}{lccc}
\hline & Average harmonicity & Smallest harmonicity Largest harmonicity \\
\hline mesh1 & 0.997292 & 0.738332 & 1.263884 \\
mesh2 & 0.997050 & 0.724865 & 1.231922 \\
mesh3 & 0.997709 & 0.755730 & 1.239204 \\
mesh4 & 0.997353 & 0.745304 & 1.270894 \\
\hline
\end{tabular}

We have a discrete mean value property if $\rho(A)=\rho_{\text {mean }}(A)$. We define the harmonicity of a node $A$ to be the ratio $\xi(A):=\rho(A) / \rho_{\text {mean }}(A)$. If the value of the harmonicity approaches the unity, the discrete mean value property is valid. We have computed the average harmonicity of the four meshes and the results can be found in the next table. As one can note in Table 2, the mesh sizes in our tests practically satisfy good harmonicity. That can equally be observed in Fig. 3 . 
Most mesh generation approaches do not take harmonicity into consideration. For the sake of comparison, we have generated four meshes for the same CAD data but with another meshing technique where the harmonicity is neglected. The resulting average harmonicities are respectively $1.603,1.909,1.512$ and 1.802. That means that neighboring edges are not guaranteed to have proportional edge size.

\section{Conclusion and Future Work}

We have described a method for generating a triangular surface mesh on a CAD model which is given by an IGES format. Neighboring edges have proportional lengths. That was achieved by imposing that the edge size function is conform with the Laplace-Beltrami operator. In the future, we will improve the implementation by including more IGES entities. Since many people move now from the graphic standard IGES to STEP, we intend also to accept STEP files. Additionally, we will develop quadrilateral meshes since they are appropriate for some graphical or numerical tasks.

\section{References}

1. Borouchaki, H., George, P.: Aspects of 2-D Delaunay Mesh Generation. Int. J. Numer. Methods Eng. 40(11), 1957-1975 (1997)

2. Bossen, F., Heckbert, P.: A Pliant Method for Anisotropic Mesh Generation. In: 5th International Meshing Roundtable Sandia National Laboratories, pp. 63-76 (1996)

3. Brunnett, G.: Geometric Design with Trimmed Surfaces. Computing Supplementum 10, 101-115 (1995)

4. Edelsbrunner, H., Tan, T.: An Upper Bound for Conforming Delaunay Triangulations. Discrete Comput. Geom. 10, 197-213 (1993)

5. Farin, G.: Curves and Surfaces for Computer Aided Geometric Design: A Practical Guide. Academic Press, Boston (1997)

6. Frey, P., Borouchaki, H.: Surface Mesh Quality Evaluation. Int. J. Numer. Methods Eng. 45(1), 101-118 (1999)

7. Graham, I., Hackbusch, W., Sauter, S.: Discrete Boundary Element Methods on General Meshes in 3D. Numer. Math. 86(1), 103-137 (2000)

8. Kolingerova, I.: Genetic Approach to Triangulations. In: 4th International Conference on Computer Graphics and Artificial Intelligence, France, pp. 11-23 (2000)

9. O'Rourke, J.: Computational Geometry in C. Cambridge Univ. Press, Cambridge (1998)

10. Randrianarivony, M.: Geometric Processing of CAD Data and Meshes as Input of Integral Equation Solvers. PhD thesis, Technische Universität Chemnitz (2006) 